\title{
Sphenoparietal Sinus
}

National Cancer Institute

\section{Source}

National Cancer Institute. Sphenoparietal Sinus. NCI Thesaurus. Code C33585.

A dural venous sinus located along the posteroinferior ridge of the lesser wing of the sphenoid bone, which drains into the cavernous sinus and receives tributaries from the superficial middle cerebral vein, veins from the temporal lobe, the anterior temporal diploic vein, and, sometimes, the frontal ramus of the middle meningeal vein. 